Trauma Berufskrankh 2004 - 6 [Suppl 3] : S291-S292 DOl 10.1007/s10039-004-0892-7

Online publiziert: 17. Juni 2004

(c) Springer-Verlag 2004
R.Winter · Gesundheitsamt Bochum, Bochum

\section{Hygiene im Operationsbereich}

\section{Rechtliche Grundlagen mit dem Schwerpunkt RKI-Richtlinie}

$A$ nforderungen an die Hygiene im Operationsbereich beruhen auf Bundes- und Landesgesetzen, Verordnungen sowie Richtlinien und Empfehlungen. Zu unterscheiden sind verbindliche von nicht verbindlichen Regelungen.

\section{Verbindlichkeit von Regelungen}

Verbindliche Regelungen müssen befolgt werden, bei Nichteinhaltung sind Sanktionen möglich (Bußgeld). Hierzu zählen Gesetze und Verordnungen, aber auch die Unfallverhütungsvorschriften, die nach $\$ 15$ des VII. Sozialgesetzbuches unmittelbar geltendes Recht darstellen.

Es gibt auch „halb verbindliche“ $\mathrm{Re}$ gelungen, diese sollen befolgt werden. Als Beispiele sind Richtlinien zu nennen. Richtlinien sind Regelungen des Handelns bzw. Unterlassens, die von einer rechtlich legitimierten Institution konsensiert, schriftlich fixiert und veröffentlicht werden.

Nicht verbindliche Regelungen können (sollten) befolgt werden. Dies gilt für Leitlinien und Standards. Leitlinien sind systematisch entwickelte Empfehlungen, die Entscheidungen über eine im Einzelfall angemessene gesundheitliche Versorgung ermöglichen sollen. Standards sind minimal akzeptable Versorgungsprozesse bzw. Ergebnisse.

Nachfolgend werden einige relevante rechtliche Grundlagen für die Hygiene im Operationsbereich aufgeführt.

\section{Verbindliche Regelungen}

Als Bundesgesetz steht das Infektionsschutzgesetz in seiner Wertigkeit über anderen rechtlichen Regelungen. Nach $\$ 23$ werden die Leiter von Krankenhäusern und Einrichtungen für ambulantes Operieren verpflichtet, nosokomiale Infektionen in gesonderter Niederschrift fortlaufend aufzuzeichnen und zu bewerten. Das gilt auch für Krankheitserreger mit speziellen Resistenzen und Multiresistenzen (MRSA!). Das Infektionsschutzgesetz legt im Übrigen fest, dass am Robert Koch-Institut eine Kommission für Krankenhaushygiene und Infektionsprävention eingerichtet wird. Diese hat die Aufgabe, Empfehlungen für Krankenhäuser und andere medizinische Einrichtungen zur Prävention nosokomialer Infektionen wie auch für organisatorische und bauliche Maßnahmen der Hygiene zu erstellen (s. „RKI-Richtlinie“).

Das Krankenhausgesetz für Nordrhein-Westfalen legt in $\$ 8$ fest, dass das Krankenhaus die erforderlichen Maßnahmen zur Erkennung, Verhütung und Bekämpfung von Krankenhausinfektionen zu treffen habe.

Auf diesem Krankenhausgesetz fußt die Krankenhaushygiene-Verordnung NRW. In $\$ 2$ dieser Verordnung wird der Krankenhausträger verpflichtet, die organisatorischen und personellen Voraussetzungen für die Einhaltung der Grundsätze der Asepsis sicherzustellen. Hiermit ist auch die Durchführung notwendiger hygienischer Maßnahmen verbunden. Diese Verordnung legt $\mathrm{u}$. a. die Bildung einer Hygienekommission fest, ferner die Ernennung eines Hygienebeauftragten sowie einer Hygienefachkraft. Aufgaben sind u. a. die Aufstellung eines Hygieneplans und die Überwachung seiner Einhaltung sowie die Erforschung von Infektionsketten und Infektionsursachen. Nach $₫ 8$ sind Art und Anzahl aller im Krankenhaus erworbenen Infektionen von der Hygienefachkraft aufzuzeichnen. Insbesondere sind in allen operativen Fachdisziplinen Sekundärheilungen statistisch zu erfassen.

\section{Nicht verbindliche Regelungen: RKI-Richtlinie}

Die sog. „RKI-Richtlinie“ der Kommission für Krankenhaushygiene und Infektionsprävention ist nach Einschätzung von juristischen Sachverständigen lediglich eine „Leitlinie“. Damit handelt es sich um eine nicht verbindliche Regelung.

Im Falle eines Kunstfehlers kann die Einhaltung einer Leitlinie jedoch als Nachweis dienen, dass die erforderliche Sorgfalt beachtet wurde und medizinische Standarderkenntnisse angewandt wurden. Somit ist die RKI-Richtlinie zwar rechtlich nicht verbindlich, schafft in diesem Sinn aber trotzdem Rechtssicherheit. Außerdem neigen Gerichte dazu, den Aussagen der RKI-Richtlinie den Charakter 
Trauma Berufskrankh

$2004 \cdot 6$ [Suppl 3] : S291-S292

DOI 10.1007/s10039-004-0892-7

○) Springer-Verlag 2004

\section{R. Winter}

\section{Hygiene im Operationsbereich. Rechtliche Grundlagen mit dem Schwerpunkt RKI-Richtlinie}

\section{Zusammenfassung}

Nach dem Infektionsschutz müssen Infektionen im Rahmen von Operationen separat aufgezeichnet und bewertet werden. Hierfür ist im Krankenhaus u. a. die Einrichtung einer Hygienekommission erforderlich. Diese muss einen Hygieneplan aufstellen. Die in der RKI-Richtlinie definierten Anforderungen der Hygiene sollten (nicht: müssen) eingehalten werden.

\section{Schlüsselwörter}

Hygiene · RKI-Richtlinie · Infektionsschutzgesetz

\section{Hygiene in surgery. Legal basis with focus on the RKI guidelines}

\begin{abstract}
According to the German Infectious Disease Control Act, infections in surgery have to be listed separately and assessed. Therefore, hospitals have to install a commission for hygiene. The commission has to set up a plan for hygiene. The requirements of hygiene listed in the RKI guidelines should be (not have to be) observed.
\end{abstract}

\section{Keywords}

Hygiene - RKI (Robert Koch Institute) guidelines . Infectious Disease Control Act von „Kunstregeln“ zuzuerkennen. Damit erhält diese die Funktion eines vorweggenommenen Sachverständigengutachtens.

Die Kommission für Krankenhaushygiene und Infektionsprävention hat $u$. a. Anforderungen der Hygiene bei Operationen und anderen invasiven Eingriffen festgelegt. Im Folgenden werden schlaglichtartig einige wesentliche Aspekte benannt.

\section{Bauliche Anforderungen nach der RKI-Richtlinie}

- Für ambulante und stationäre Eingriffe gelten jeweils die gleichen Anforderungen der Hygiene.

- Für den Operationsraum gilt, dass keine Wandarmaturen sowie Bodeneinläufe vorhanden sein dürfen. Die Ein- bzw. Ausleitung ist ggf. im Operationsraum selbst möglich. Ein Waschraum kann für 2 nebeneinander liegende Operationsräume genutzt werden.

- Die Personalumkleide hat eine unreine Seite mit Schränken für die Straßenkleidung, außerdem Waschbecken/WC, ggf. Duschen. Hier befindet sich ein Sammelbehälter für benutzte OP-Kleidung. Die reine Seite verfügt über ein Lager für den Tagesbedarf der reinen Bereichskleidung. Der Weg vom zentralen OP-Gang führt direkt zurück zur unreinen Seite der Personalumkleide.

- Die Patientenübergabe verfügt über eine unreine und reine Seite. Der Aufwachraum liegt „am Übergang“ von der OP-Abteilung zum übrigen Krankenhaus. Weitere Räume sind das Lager für saubere Geräte bzw. Sterilgut, Materialversorgung, der unreine Arbeitsraum (zur Aufbereitung unreiner bzw. benutzter Geräte), der Entsorgungsraum für unreine Güter, Raum für Putzmittel sowie Aufenthaltsraum für Mitarbeiter.

\section{Organisatorische Anforderungen nach der RKI-Richtlinie}

- Es sollen sich so wenig Personen wie möglich im Operationsraum aufhalten. Die Türen des Operationsraums bleiben soweit möglich geschlossen.
- Nach jeder Operation ist eine desinfizierende Reinigung der patientennahen Flächen erforderlich. Das gilt auch für den gesamten begangenen Fußboden des Operationsraums sowie für sichtbare Verunreinigungen.

- Eine hygienische Händedesinfektion ist erforderlich vor Anlegen der keimarmen Bereichskleidung (in der Personalumkleide), vor Verlassen der Personalumkleide sowie nach Toilettenbesuch. Mund-/Nasenschutz ist vor Betreten des Operationsraums anzulegen und muss für jede Operation sowie bei Verschmutzung und Durchfeuchtung erneuert werden. Nach der chirurgischen Händedesinfektion werden im Operationsraum der sterile Operationskittel und anschließend sterile Handschuhe angelegt.

\section{Korrespondierender Autor Dr. R. Winter}

Gesundheitsamt Bochum, Westring 28/30,44777 Bochum, E-Mail:winter@bochum.de

Interessenkonflikt: Der korrespondierende Autor versichert, dass keine Verbindungen mit einer Firma, deren Produkt in dem Artikel genannt ist, oder einer Firma, die ein Konkurrenzprodukt vertreibt, bestehen. 